\title{
Introduction
}

Les années 2000 - une révolution "française" pour Beckett?

\author{
Matthieu Protin \\ Institut de Recherches en Études Théâtrales de la Sorbonne Nouvelle, \\ Paris 3, Paris, France \\ matthieuprotin@gmail.com
}

Longtemps la mise en scène de Beckett en France fut nietzschéenne-placée sous le signe de "l' éternel retour", comme s'il n' existait qu'une seule façon de monter ses pièces, celle initiée en 1953 dans la petite salle du théâtre de Babylone. Praticiennes et praticiens se trouvaient, d'une certaine façon, pris entre deux feux: d'un côté, des didascalies aux allures de partition, statut encore renforcé par les contrats et les obligations liées aux droits d' auteur; de l' autre, une tradition solidement établie, par Blin, d'abord, puis autour de deux grandes figures du théâtre français, Jean-Louis Barrault et Madeleine Renaud. Dès lors, comment s'étonner que la majeure partie des mises en scène perpétuent inlassablement le modèle "Blin"? Décors minimaux, aucune transposition ou décalage, et, surtout, pas d'interprétation trop poussée qui donnerait le sentiment de s'écarter du Beckett canonique, traditionnel. Cet effet est encore renforcé, en France, par l'absence de toute édition critique ${ }^{1}$ de ses textes, alors que présenter, par exemple, les variantes, les coupes et les réécritures montreraient un autre Beckett, et surtout une autre façon d' appréhender ses célèbres didascalies. Alors, bien sûr, il y eût, ici ou là, des tentatives: le traitement délibérément clownesque de Marcel Maréchal pour Fin de partie; l' audace, finalement punie, de Gildas Bourdet, pour cette même pièce, à la Comédie Française; le traitement profondément social et référentiel que proposa Jouanneau d'En attendant Godot. Mais ces traitements singuliers restèrent, justement, comme des "anomalies", des écarts. Ne saurait-il être question de traiter Beckett comme un

1 Il nous semble ici important de souligner la responsabilité des Editions de Minuit et d'Irène Lindon dans cette situation. Derrière l'intention, tout à fait honorable, de préservation de l'œuvre de Beckett, on assiste en définitive à un risque de fossilisation qui se prolonge aussi au plateau, avec une interprétation très rigoriste des préceptes de Beckett, en contradiction avec sa propre attitude vis-à-vis de la scène, qui évolua tout au long de sa vie. 
autre auteur? Comme si ses pièces se dérobaient, au fond, et avec la volonté indéniable de l' auteur, à tout traitement trop "affirmé". Ce n' est d' ailleurs pas un hasard si, parmi les grands noms de la mise en scène en France dans les années 1970-1980, nombreux furent celles et ceux qui reconnurent, à l'instar de Vitez, ne pas trop savoir ce qu' ils pourraient apporter à ce que Blin avait fait... Mettre en scène soumettait inexorablement à la menace du "déjà-vu".

Cela ne signifie nullement que le travail de celles et de ceux qui entreprirent de monter les pièces de Samuel Beckett avant les années 2000 ne présentait pas d' intérêt. Simplement, il y avait, malgré tout, le sentiment que le traitement proposé de Beckett sur une scène française qui, pourtant, se singularisa très tôt par l' audace de son traitement des classiques et qui peut s'enorgueillir de compter, dans ses rangs, des pionniers importants de la mise en scène moderne, restait, au fond, en retrait. Face à l'œuvre de Beckett, praticiennes et praticiens succombaient à un double retour vers le passé: passé d'une mise en scène canonique, celle de Blin, qui inaugura un traitement spécifique de l'œuvre beckettienne qui fonctionna longtemps comme une persistance rétinienne; mais passé, aussi, de la mise en scène elle-même, retour vers une époque, avant Craig, avant Appia, avant Antoine et Lugné-Poe. Une époque, au fond, où la mise en scène cesse d'être cet art moderne qui postule une ambition herméneutique, une création autre, par rapport au texte écrit, pour n'être plus que simple concrétisation. Face à l'œuvre beckettienne, sur la scène française, la double souveraineté caractéristique des années 1950 se trouvait subitement abolie: seul régnait "Sire le mot", pour reprendre une expression de Gaston Baty. Un contraste d' autant plus saisissant, quand, par hasard, une mise en scène allemande venait s' égarer sur les planches francophones. Et l'un des auteurs de ces lignes se souvient de l'étonnement qui l' avait saisi à la vue d' un Endspiel où Hamm était joué par un enfant... "ainsi, un autre Beckett était possible?!" Telle fut la pensée qui surgit au moment même où le drap fut enlevé... Oui, un autre Beckett était possible. Les années 2000 se chargeront de le prouver.

Aborder Beckett sur la scène française dans les années 2000, c'est donc se trouver au cœur d'une tension entre rupture et continuité. Continuité, tout d'abord, notamment avec l'intégrale Beckett qui fut présentée en 2006, à l' occasion du centenaire de la naissance de l' auteur; mais rupture, déjà, dans cette même intégrale, avec, par exemple, le traitement marionnettique d'Acte sans Parole 1, proposé par François Lazaro et Aurélia Ivan. Oui, peu à peu, la relation avec l'œuvre beckettienne s' assouplit. On osa davantage. Peut-être parce que cette intégrale venait aussi, d'une certaine façon, clôturer le traitement proposé depuis les années 1950: comme un résumé, qui viendrait indiquer, aussi, la nécessité de passer à autre chose, à une autre conception, et d'aller puiser, en particulier à l' étranger, une autre façon de faire. Il n' est ainsi pas ano- 
din, quand on regarde les mises en scène de Beckett en France, d' observer que les montages, toujours délicats à réaliser car nécessitant des autorisations très spécifiques, n' apparaissent qu'à partir de 2008: d'abord un Triptyque Beckett, par Moni Grego, à partir des dramaticules, puis Chantiers Beckett de Katia Hernandez, en 2014, et enfin Juste le temps d' après Samuel Beckett, mis en scène par Bruno Meyssat en 2017. De tels spectacles, procédant par montages, voire "d'après" Beckett, viennent alors témoigner de la façon dont, comme le soulignait Stanley Gontarski, l' année 2006 marque l'entrée de Beckett dans une nouvelle ère (30). À partir de l' année 2006 le traitement scénique des œuvres de Beckett va commencer à évoluer. La France, une fois n'est pas coutume, entame, fort tardivement, sa révolution. Trois phénomènes en témoignent, tout particulièrement, sur lesquels reviendront les différents articles de ce numéro de Samuel Beckett Today/Aujourd'hui.

Tout d'abord, des figures importantes du paysage théâtral français, comme Jean-Pierre Vincent, qui fut administrateur de la Comédie-Française, et Alain Françon - qui resta plusieurs années à la tête du Théâtre National de la Colline où il entama une collaboration particulièrement fructueuse avec Edward Bond - prirent pour Beckett, dans les années 200o, un intérêt nouveau. Des interprètes majeurs, aussi, comme Denis Lavant, ou Catherine Frot. Cet intérêt est tout sauf anecdotique: il marque la fin d'un certain ostracisme envers Beckett, bien établi dans le paysage théâtral français, depuis les années 1950. En effet, le modèle de l'engagement, dans la littérature comme dans l'art, jouait un rôle fondamental, auquel il n'était pas question que le théâtre échappât. Dès lors, entre deux B, il fallut choisir: on était Brecht ou Beckett, dans les années 196o-1970, comme on était Rolling Stones ou Beatles. L'opposition était forte, comme le rappelle Jeanyves Guérin (42), et comme en témoignent, par exemple, les articles d'un Roland Barthes qui accusa le théâtre de Beckett d' être "bourgeois", adjectif particulièrement stigmatisant. Beckett lui-même ne fera rien pour lever ce malentendu. Il l'approfondira, même, par son apparent désengagement, tant dans ses paroles publiques - que l'on songe à la célèbre lettre à Michel Polac - que dans son processus d'écriture, tel que l'analyse, par exemple, Stanley Gontarski, et qui voit toutes les références historiques s'estomper au fur et à mesure que l'œuvre prend forme. Cet ostracisme n' est pas de l'histoire ancienne, dans les années 200o, comme en témoigne, par exemple, la comparaison établie par Georges Banu entre Beckett et Brecht, et qui tourne, forcément, à l' avantage du second:

Par ailleurs, le malentendu [entre auteur et metteur en scène] peut être suspendu quand les deux fonctions se confondent en un seul artiste, sans que, chez lui, l'une soit en retard sur l'autre: ce fut le cas de Brecht 
auteur/metteur en scène de ses pièces et nullement de Beckett. On peut s'en réjouir pour le succès de l'un et déplorer l'échec de l' autre.

BANU, 242

Ensuite, une ère nouvelle s'ouvre: en témoigne le non-respect d'un certain nombre de traits caractéristiques de ce que l'on pourrait appeler le traitement "conventionnel" de Beckett. Ainsi, avec Catherine Frot, la singularité du traitement français de Winnie, le non-respect, qui, apparemment, ne choquait cette fois personne, des didascalies initiales, est rompu. Oh les beaux jours cesse d'être une pièce sur la vieillesse. Ce que toute personne - britannique ou non ayant vu la mise en scène de Beckett par Beckett à Londres avec Billie Whitelaw dans le rôle-titre savait bien se révèle à présent dans la scène française. Le traitement par la danse et le cirque d'Acte sans parole par Dominique Dupuis inaugure une hybridité, un mélange des genres envers lesquels Beckett se montra longtemps rétif. L'interprétation ouvertement politique de Godot proposée par Malaguerra, Lambert-Wild et Bozonnet, rompt avec un parti pris de neutralité qui, pour respectueux qu'il fût des intentions de Beckett, ne pouvait durer trop longtemps sans risquer de livrer à la sclérose une œuvre absolument majeure.

Enfin, l'ouverture de la scène française à des mises en scène venues de l'étranger, contribua fortement à renouveler l' appréhension de Beckett dans le pays qui le vit accéder à la gloire littéraire, mais qui, de manière symptomatique, ne le vit que très rarement assumer la fonction de metteur en scène. Certains des spectacles les plus marquants sont ainsi l'œuvre d' un Bob Wilson ou d' une Tania Bruguera, et nul doute que leur traitement, par leur originalité, par une certaine tendance à être fidèlement infidèles aussi, dessine une voie féconde pour une scène qui n' avait pas, par exemple, connu le traitement de Fin de partie par une JoAnne Akalaitis.

Rupture avec la mise en scène canonique de Blin; fin de l'ostracisme pour des raisons politiques; découvertes de traitements peu orthodoxes des œuvres beckettiennes; tout cela va de pair avec un autre changement majeur, porté, non seulement par des artistes, mais aussi par des chercheuses et des chercheurs: la prise en compte, encore timide, mais néanmoins bien réelle, de la mouvance de l'œuvre beckettienne, sous l'influence de la scène. Longtemps caractérisée par un certain "texto-centrisme", la mise en scène de Beckett en France allait peu à peu sortir des sentiers battus, pour découvrir, par-delà les sacro-saintes didascalies, d' autres manières de faire, de vivre, et de voir Beckett.

Voilà pourquoi ce numéro fut envisagé comme un dialogue, avec la scène, à travers des analyses de chercheuses et de chercheurs, d'une part, et des entretiens avec des praticiennes et des praticiens, d'autre part. Il ne s'agit pas de 
prendre la parole des artistes comme une parole indiscutable et indiscutée. On peut par exemple constater que si certains metteurs en scène reprennent l'idée du texte de Beckett comme partition, ce respect revendiqué ne rend pas compte des innovations de la mise en scène, tout comme $a$ contrario des propos de rupture peuvent recouvrir une mise en scène beaucoup plus "conventionnelle". Notre ambition, plus modeste, est de voir dans ces entretiens le témoignage d' une nouvelle façon d'envisager le rapport à l'œuvre de Beckett. Nous avons essayé, bien sûr, de maintenir un certain équilibre, et nous espérons que cette alternance, permettant à la fois de comprendre comment l' œuvre se fait, et comment elle se perçoit dans une histoire plus longue, permettra de mieux saisir le rôle considérable joué par les années 2000, jusqu'à aujourd'hui, dans le traitement de Beckett sur la scène qui l'a vu naître, en tant qu' auteur dramatique. Le mouvement est encore en marche, et la révolution est loin d'être terminée. Sans doute, pour s' accentuer, faudrait-il qu' elle s' accompagne aussi de nouvelles éditions, plus critiques, donnant à voir et à comprendre la plasticité de cette écriture, et les évolutions, les doutes, les repentirs de son auteur. Voilà pourquoi ce numéro n'a aucunement la prétention d'être une "somme" qui viendrait définitivement clôturer la question. Au contraire: il propose d' ouvrir un champ de recherche encore vaste, en fournissant des appuis, des repères, mais en étant, aussi, très conscient de ses manques. Après tout, comment oublier que le gris domine dans l'univers beckettien? Tout n' est pas noir ou blanc - innovant ou traditionnel. Les deux se mêlent souvent, y compris dans les propos, où reviennent, même pour des mises en scène qui se placent sous le signe d' une indéniable rupture, l' idée d'un sacro-saint respect des didascalies. Toute révolution se place dans une tension entre ces deux pôles, entre rupture et continuité, et celle détaillée dans ces pages n'échappe pas à la règle.

Quelques remarques, enfin, avant de laisser Marie-Claude Hubert procéder à une vision rétrospective de la mise en scène de Beckett en France.

On nous a fait remarquer que notre numéro laissait peu de place aux femmes - en particulier aux artistes. La rigidité de Beckett sur ce point, qui opposait l'absence de prostate à toute mise en scène de Godot avec des femmes - étrange souci du réalisme pour un auteur d'ordinaire peu soucieux de telles considérations - avait-elle déteint sur celles et ceux qui l' étudiaient? Sans doute faut-il davantage y voir un phénomène générationnel. Quand on regarde les mises en scène les plus marquantes, celles-ci correspondent nous l'avons dit à des hommes ayant commencé leur carrière théâtrale dans les années 1970, à une époque où les femmes étaient alors minoritaires en tant que metteuses en scène, et les mises en scène de Beckett en France restent souvent l'apanage des metteurs en scène. Cela ne signifie nullement qu'il n'y a pas d'exception, le travail de Stéphanie Chévara autour de la création 
de Godot ${ }^{2}$ témoigne aussi de la prise en compte des documents alentours, et d'un regard, quasiment génétique sur l'œuvre. Il n' en reste pas moins que des artistes majeures de la scène française ${ }^{3}$ ne s' emparent pas de Beckett, étant bien davantage dans des pratiques d'écriture de plateau et de collectif...

Le second point sur lequel nous voudrions conclure, est le caractère un peu trompeur de ce titre: après tout, nous allons bien au-delà des années 2000... alors, nous pourrions déclarer qu' en définitive nous traitons les années 2006-2016, mais que cela "sonnait drôlement" comme dirait Camier. Cependant il nous semble véritablement important d' envisager cette évolution dans un temps long, dont nous faisons, encore aujourd' hui, partie. D' une part parce que pour avoir fait travailler des étudiants sur Fin de partie en temps de pandémie, je crois que la pièce leur semble tout à fait concrète, et nullement absurde. D' autre part, parce que la crise a empêché la tenue d'un certain nombre de mises en scène, par exemple un Godot mis en scène par Françon, qui auraient témoigné de la permanence des phénomènes mentionnés dans ce numéro.

Enfin, sans doute manque-t-il encore parfois une vision plus panoramique sur ces années. Nous avons, chaque fois, essayé de faire en sorte que le cas particulier vienne se tisser avec des enjeux généraux. Cependant nous n' oublions pas que notre titre parle de la scène, qui est avant tout, un espace concret. Comme le rapportait plaisamment Denis Guénoun, alors qu'il réfléchissait à une ontologie de la scène, il interrogea le chef-machiniste de la Comédie de Reims, Jean-Luc Toussaint. Ce dernier lui répliqua: "Si on ne peut pas y planter des clous, ce n'est pas une scène." (22) Voilà pourquoi il nous a semblé qu' aborder Beckett sur la scène française devait se faire depuis le plateau, ce qui, ici encore, marque une rupture avec une certaine façon de voir Beckett en France. Après tout, pour que l'éternel retour que nous évoquions puisse avoir lieu, il faut bien s' en tenir à la page. La scène, elle, offre sans cesse le spectacle de son incessante métamorphose, que ce numéro s'efforce, à sa manière, de fixer un instant.

\section{Ouvrages cités}

Banu, Georges, "L'auteur et le metteur en scène, un malentendu qui dure", dans Avènement de la mise en scène/Crise du drame. Continuités-discontinuités, actes de la

2 Stéphanie Chévara, Naissance d'un chef d'œuvre, spectacle qui tourna notamment en 2017.

3 Citons, sans exhaustivité aucune, Jeanne Candel, Anne Monfort ou Mathilde Delahaye, qui sont toutes dans une esthétique plus post-dramatique, du côté des écritures de plateau et des créations collectives. 
rencontre franco-italienne Paris, Théâtre de la Colline, 12-13 décembre 2008, Jean-Pierre Sarrazac et Marco Consolini (dir.) (Bari, Edizioni di Pagina, 2009), 237244.

Gontarski, Stanley, "Beckett en son époque / Beckett en notre époque", Registres, $\mathrm{n}^{\mathrm{o}_{22}}$ (Paris, Presses de la Sorbonne Nouvelle, Printemps/Été 2020), 29-41.

Guénoun, Denis, "Qu'est-ce qu'une scène?", dans Philosophie de la scène (Besançon, Les Solitaires intempestifs, 2010), 11-24.

Guérin, Jeanyves, "Lectures politiques d'En attendant Godot", Registres, no 22 (Paris, Presses de la Sorbonne Nouvelle, Printemps/Été 2020), 42-55.

\section{Appendix: Liste des principales mises en scène des œuvres de Beckett en France depuis 2000}

Sources: Archives du spectacle; $\mathrm{CNT}$

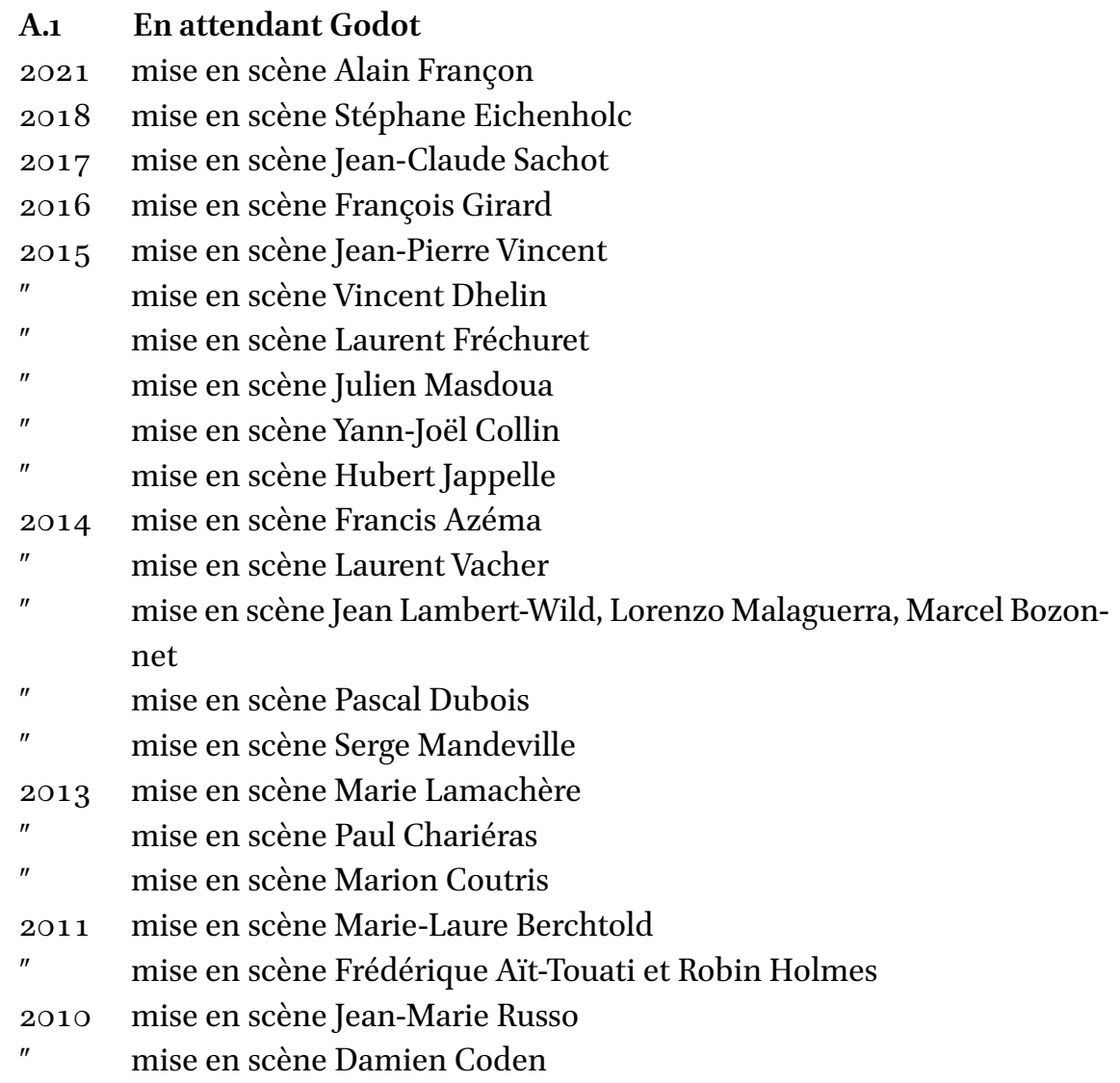




\begin{tabular}{|c|c|}
\hline " & mise en scène Julie Loubère \\
\hline " & mise en scène Hans-Peter Cloos \\
\hline 2009 & mise en scène Bernard Lévy \\
\hline " & mise en scène Elvire Brison \\
\hline 2008 & mise en scène Patrick Reynard \\
\hline 2007 & mise en scène René Chéneaux \\
\hline$"$ & mise en scène Hassane Kouyaté \\
\hline 2003 & mise en scène Lucie Gougat \\
\hline$"$ & mise en scène Leslaw Janicki \\
\hline 2002 & mise en scène Bernard Sobel \\
\hline 2001 & mise en scène Gilles Bouillon \\
\hline
\end{tabular}

\section{A.2 Fin de partie}

2019 mise en scène Vincent Dhelin

" mise en scène Didier Viéville

2018 mise en scène Fred Tournaire

2017 mise en scène Tania Bruguera

2015 mise en scène Pascal Dubois

" mise en scène Sandrine Bauer

" mise en scène Jean-Claude Sachot

2014 mise en scène Virgile Simon

2012 mise en scène Kenza Jernite

2011 mise en scène Thomas Simet

" mise en scène Alain Françon

" mise en scène Yves Borrini

2010 mise en scène Krystian Lupa

" mise en scène Yvan Blanloeil

" mise en scène Éric Sanjou

" mise en scène Jean Pétrement

2009 mise en scène Yves Borrini

2008 mise en scène Charles Berling

2006 mise en scène Bernard Lévy

2005 mise en scène Alain Timár

" mise en scène Béatrice de La Boulaye

2002 mise en scène Laurent Maindon

2001 mise en scène Gilles Bouillon

" mise en scène Christian Colin

2000 mise en scène Jean-Claude Fall 


$\begin{array}{ll}\text { A.3 } & \text { La derniere bande } \\ 2019 & \text { mise en scène Jacques Osinski } \\ 2017 & \text { mise en scène Dan Jemmett } \\ 2016 & \text { mise en scène Peter Stein } \\ " & \text { mise en scène Cécile Gheerbrant } \\ 2012 & \text { mise en scène Alain Françon } \\ 2011 & \text { mise en scène Hubert Jappelle... } \\ 2010 & \text { mise en scène Christophe Gand } \\ " & \text { mise en scène Jean Le Scouarnec } \\ 2009 & \text { mise en scène Robert Wilson } \\ 2008 & \text { mise en scène Christophe Perton } \\ 2007 & \text { mise en scène Charles Lee } \\ " & \text { mise en scène Olivier Bénézech } \\ " & \text { mise en scène Pierre Chabert } \\ 2006 & \text { mise en scène Xavier Marchand } \\ 2005 & \text { mise en scène José Manuel Cano Lopez } \\ " & \text { mise en scène Éric Zobel } \\ \text { " } & \text { mise en scène Alain Milianti } \\ 2004 & \text { mise en scène Isabelle Luccioni } \\ 2002 & \text { mise en scène Diden Berramdane } \\ 2000 & \text { mise en scène Pierre Vincent }\end{array}$

\section{A.4 Oh les beaux jours}

2021 mise en scène Claude Vuillemin

2019 mise en scène Mathilde Heizmann

2018 mise en scène Michael Delaunoy

2017 mise en scène Isabelle Martinez...

2015 mise en scène Pascal Dubois

2014 mise en scène Stéphane Braunschweig

" mise en scène Anne Bisang

2013 mise en scène Serge Noyelle

" mise en scène Rush Rehm

2012 mise en scène Marc Paquien

2011 mise en scène Michel Abécassis

" mise en scène Blandine Savetier

" mise en scène Catherine Barbier

2010 mise en scène Daniel Proïa...

" mise en scène Maria Naudin

2009 mise en scène Yves Borrini

" mise en scène Jean-Luc Terrade 


$\begin{array}{ll}" & \begin{array}{l}\text { mise en scène Robert Wilson } \\ \text { mise en scène André Brassard } \\ "\end{array} \\ 2007 & \begin{array}{l}\text { mise en scène Jean-Pierre Brière } \\ \text { mise en scène Deborah Warner }\end{array} \\ 2003 & \text { mise en scène Arthur Nauzyciel } \\ 2001 & \text { mise en scène Laurent Fréchuret } \\ 2000 & \text { mise en scène Joël Jouanneau }\end{array}$

\section{A.5 Comédie}

2020 Comédie / Wry Smile Dry Sob d'après Samuel Beckett... mise en scène Silvia Costa

2018 mise en scène Sahika Tekand

2014 mise en scène Sarah Mouline

2011 mise en scène Jacqueline Gudin

2009 mise en scène Magdalena Czartoryjska-Meier

2004 mise en scène Isabelle Luccioni

\section{A.6 Acte sans paroles 1}

2013 mise en scène Dominique Dupuy

" mise en scène Stéphane Fortin

2011 mise en scène Zoran Petrovic

2006 mise en scène Aurelia Ivan et François Lazaro

\section{A.7 Dramaticules, Pièces courtes, Pieces rassemblées}

2021 Words and Music, mise en scène Jacques Osinski

2019 Pas, mise en scène Jane Friedrich...

2016 Abîmés (Quoi où, Souffle, Impromptu d'Ohio et Pas), mise en scène Catherine Bourgeois

2014 L'Impromptu d'Ohio, mise en scène Vincent Lacoste

" Not I, Footfalls et Rockaby, mise en scène Walter Asmus

" Cette fois, mise en scène Danielle Bré

2013 Cette fois, mise en scène Vincent Lacoste

2012 Tous ceux qui tombent, mise en scène Jacques Nichet

2007 Pas-Pas moi-Va-et-vient, mise en scène Yvonne McDevitt, conception Clara Simpson

" Fragments, mise en scène Peter Brook

" Pas moi, mise en scène Charles Lee

2006 Cinq dramaticules, mise en scène Jean Dautremay

" Comédie, Pas, Catastrophe, mise en scène Michael Lonsdale

" Tous ceux qui tombent, mise en scène Albert Simond 
2005 Solo et La Dernière Bande, mise en scène Alain Milianti

2004 ... que nuages... d'après Samuel Beckett, mise en scène Madeleine Louarn

2002 La Dernière Bande - Pas, mise en scène Diden Berramdane

2001 Fragments de théâtre I et II, mise en scène Annie Perret

2000 Comédie - Fragments 2 - Actes sans paroles, conception Fabienne Margarita

$\begin{array}{ll}\text { A.8 } & \text { Premier amour } \\ 2020 & \text { mise en scène Barbara Baker... } \\ 2017 & \text { mise en scène Jacques Fontaine } \\ 2016 & \text { mise en scène Vincent Auvet } \\ 2013 & \text { conception Jean-Marc Bourg } \\ 2012 & \text { mise en scène Manon Allouch } \\ 2011 & \text { mise en scène Wénaël Astier } \\ 2010 & \text { mise en scène Jean-Marie Papapietro } \\ 2009 & \text { mise en scène Sami Frey } \\ 2007 & \text { mise en scène Charles Lee } \\ 2006 & \text { mise en scène Alexandra Royan } \\ 2004 & \text { mise en scène Yves Gourmelon } \\ 2001 & \text { mise en scène Jacques David }\end{array}$

A.9 Autres textes non dramatiques

2021 L'Image, mise en scène Jacques Osinski

2018 Compagnie, mise en scène Jacques Nichet

2017 Cap au pire, mise en scène Jacques Osinski

2016 Compagnie, mise en scène Christian Gonon

" Le Dépeupleur, mise en scène Alain Françon

2013 Têtes mortes, mise en scène Marie Lamachère

2003 Le Dépeupleur, mise en scène Serge Merlin

A.10 Divers (montages, d'après etc.)

2020 Happy Daze d'après Samuel Beckett, mise en scène Aurélie Imbert

2019 Variations sur Cap au pire de Samuel Beckett, conception Daniel Erdmann

" Triptyque Beckett d' après Samuel Beckett, mise en scène Gabriel Alvarez

2018 Tu viens jouer avec moi, sinon je te mets une claque d'après Samuel Beckett, mise en scène Julie Petitrenaud

" Oh! d' après Samuel Beckett, mise en scène Virginie Marouzé 
2017 Juste le temps d'après Samuel Beckett, mise en scène Bruno Meyssat

" Le Projet Beckett de Samuel Beckett, mise en scène Diane Loiselle

" $\quad 2 k$ d'après Samuel Beckett, conception Ho Geung-Nam

" This is my last dance d' après Samuel Beckett, conception Tabea Martin

2012 Quand c'était d' après Samuel Beckett, mise en scène Katia Hernandez

" Cap d'après Samuel Beckett, conception Aurelia Ivan

2014 Chantiers Beckett, mise en scène et conception Katia Hernandez

" Un temps bis d' après Samuel Beckett, conception Georges Aperghis

" Juste une valse d' après Samuel Beckett, mise en scène Fabio Ezechiele Sforzini

2009 B. 1. Butô 2. Beckett d' après Samuel Beckett, mise en scène Philippe Lanton

2008 Triptyque Beckett, mise en scène de Moni Grego

2007 Dire Beckett d' après Samuel Beckett, mise en scène Cyril Desclés

2006 Quelques mots sur le silence de Samuel Beckett, mise en scène Marek Kedzierski conception Claire Aveline

" Nous comparerons nos blessures d' après Samuel Beckett et Ludovic Janvier, mise en scène André Le Hir

" Actes avec ou sans paroles d'après Samuel Beckett, mise en scène Sophie Loucachevsky

" Mademoiselle Julie - Pas moi d'après August Strindberg et Samuel Beckett, mise en scène Yves Ferry et Moni Grego

2000 Paroles données d' après Samuel Beckett, mise en scène Sandrine Lanno " Comédie $(s)$ d' après Samuel Beckett, mise en scène Jean-Yves Pénafiel

" Stéréo d' après Samuel Beckett, mise en scène Gwenaël Morin 